\title{
Microvascular abnormalities in Rett syndrome
}

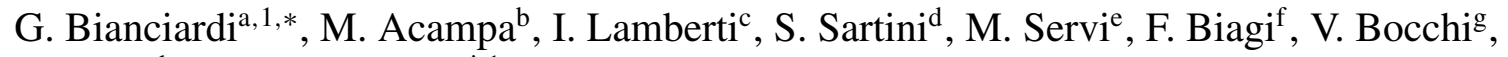 \\ J. Hayek ${ }^{\mathrm{h}}$ and M. Pastorelli $\mathrm{i}^{\mathrm{i}, 1}$ \\ a Dipartimento Biotecnologie Mediche, Anatomia Patologica, Università degli Studi di Siena, Siena, Italy \\ ${ }^{\mathrm{b}}$ Dipartimento Scienze Neurologiche e Sensoriali, Azienda Ospedaliera Universitaria Senese, Siena, Italy \\ ${ }^{\mathrm{c}}$ Emergenza Territoriale, Ospedale San Donato USL8, Arezzo, Italy \\ ${ }^{\mathrm{d}}$ Scuola di Specializzazione in Medicina D’Emergenza-Urgenza, Università degli Studi di Siena, \\ Siena, Italy \\ ${ }^{\mathrm{e}}$ Medicina Generale, Ospedale Villa Marina, Piombino, Italy \\ ${ }^{\mathrm{f}}$ Immunoematologia e Medicina Trasfusionale, USL9, Grosseto, Italy \\ ${ }^{\mathrm{g}}$ Scuola di Specializzazione in Medicina Interna, Università degli Studi di Siena, Siena, Italy \\ ${ }^{\mathrm{h}}$ Neuropsichiatria Infantile, Azienda Ospedaliera Universitaria Senese, Siena, Italy \\ ${ }^{\mathrm{i}}$ Dipartimento Medicina Clinica e Scienze Immunologiche, Università degli Studi di Siena, Siena, Italy
}

\begin{abstract}
Rett syndrome (RTT) is a post-natal neurological disorder that represents the second most common cause for mental retardation. The presence of cold hands and feet, and blue, a feature frequently observed in these patients, is one of the nonneurological phenotypes that characterizes RTT, up to now not well explained. We have performed videocapillaroscopy in subjects affected by Rett syndrome. We have observed ramified and bushy capillaries, characteristic features of neoangiogenic capillaries, dilated capillaries and an irregular and chaotic microvascular pattern. To quantify these features and to evaluate the microvascular pattern complexity, we have performed a fractal analysis. Fractal dimension and Lempel-Ziv indexes resulted higher in Rett females than in age-matched healthy females $(p<0.001 ; p<0.001)$. Our findings indicate the presence of previously unrecognized microvascular abnormalities in Rett syndrome.
\end{abstract}

Keywords: Rett syndrome, microvessels, fractal analysis, complexity analysis

To the Editors,

Rett syndrome (RTT) is a post-natal neurological disorder characterized by motor abnormalities, autistic symptoms, cognitive deficiencies and decreased brain growth during childhood [4]. With 1:10.000 live births, it represents the second most common cause for mental retardation. In up to $95 \%$ of patients (females), mutations in the transcriptional regulator X-linked gene methyl CpG-binding protein 2 (MECP2) have been described [3].

\footnotetext{
${ }^{1}$ These authors contributed equally to the study.

${ }^{*}$ Corresponding author: G. Bianciardi, Dipartimento Biotecnologie Mediche, Anatomia Patologica, Università degli Studi di Siena, Via delle Scotte 6, 53100 Siena, Italy. E-mail: giorgio.bianciardi@ unisi.it.
} 
In this pervasive developmental disorder many phenotypic alterations have been described [9]. Small hands and feet, deceleration of the rate of head growth (including microcephaly in some), growth failure and scoliosis have been described. In RTT, the autonomic nervous system resulted abnormal at various levels, from the central to the peripheral nervous system [2], alterations related to the cardiovascular system were observed, with a 300 -fold increased risk of sudden cardiac death [1], as were breathing dysfunctions [10].

Up to now, the presence of cold hands and feet, and blue, a feature frequently observed in these patients, is not well explained. To test the hypothesis of a microvascular involvement in RTT, we performed in RTT a nailfold capillaroscopy in order to characterize the microvessel morphology and to perform a complexity analysis of the microvascular pattern. The study was performed in accordance with the ethical guidelines for Clinical Hemorheology and Microcirculation (Clin Hemorheol Microcirc. 2010;44(1):1-2)

In this study we included 57 females, mean age of 9.27 years ( $\mathrm{SD}=4.5$; range $4.1-17.9$ years), with a clinical diagnosis of typical RTT based on revised criteria for this disorder [12], made in each individual by a neuropsychiatrist (J.H.) and confirmed by mutation analysis of the MECP2 gene. 47 age-matched healthy females (mean age of 10.32 years $(\mathrm{SD}=4.3$; range 5.0-17.5 years) were also enrolled. All the subjects with any pathologies able to modify the morphologic appearance of the microvascular pattern (e.g. diabetic microangiopathy, hypertension, connective tissue diseases) were excluded.

Nailfold capillaroscopy was performed with a VideoCap (DS Medica, Italy), giving a total magnification of $\times 200$. All observations were performed with the subjects in a constant temperature setting $\left(22^{\circ}\right.$ to $25^{\circ} \mathrm{C}$ ). Images of three fields for subject were stored.

Manual outline of the two-dimensional microvascular pattern's trajectories was performed by two operators who were unaware of the subject's category using Adobe Photoshop software. Non-readable areas were $<10 \%$ of the total area. Images were processed to threshold the microvessel pattern without background interference. The microvascular pattern was subsequently converted to outlines of a single pixel, using Image Pro-plus 1.3 software (Image Pro-plus Media Cybernetics Inc., Silver Spring, MD, USA) (Fig. 1).

To evaluate the geometrical complexity of the microvascular pattern the local fractal dimension. D, was measured using the box-counting algorithm, using a software written by us. Briefly, each image
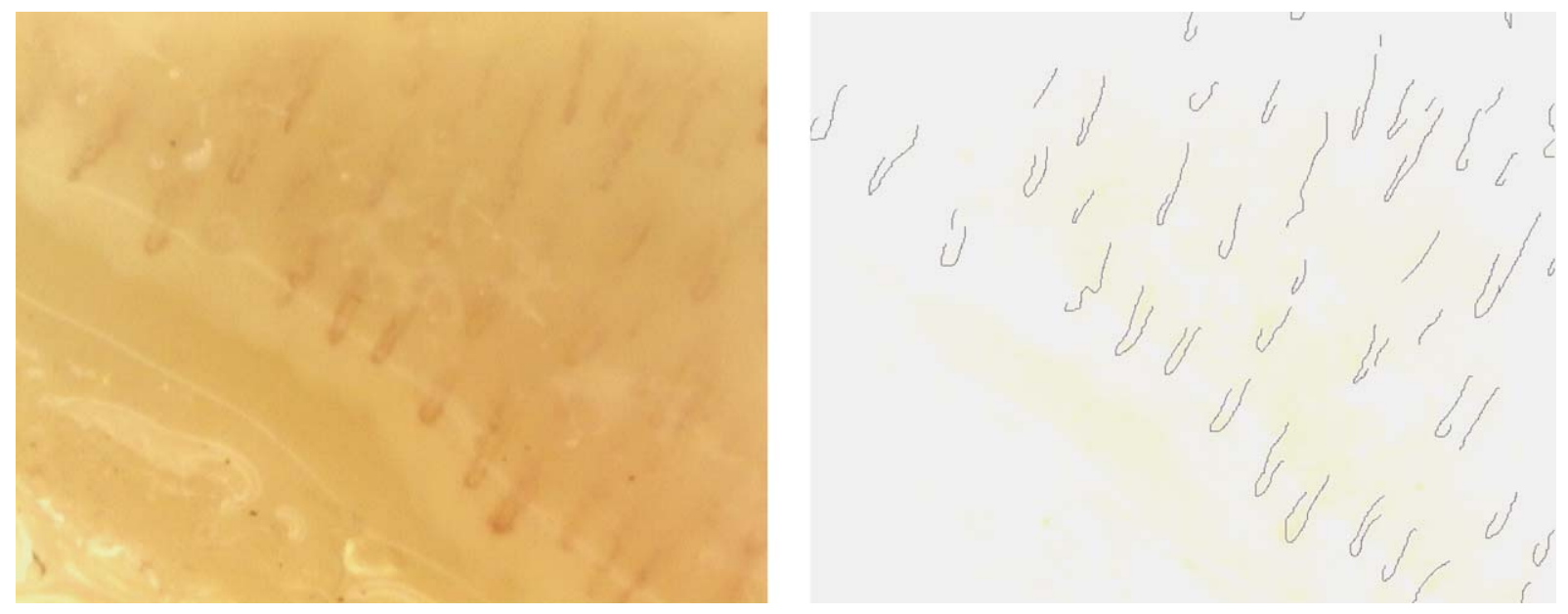

Fig. 1. Videocapillaroscopy $(\times 200)$ and thresholded image in a control subject. 
was covered by a net of L square boxes (from 1 to 130 pixels) and the number of boxes containing any part of the outline $\mathrm{Nb}(\mathrm{L})$ was counted. The slope of the $\log -\log$ plot of $\mathrm{Nb}(\mathrm{L})$ vs. 1/L represented the fractal dimension of the distribution [8]. The log-log straight line $(p<0.001)$ revealed the statistical selfsimilarity (fractal) of the microvascular pattern. The procedure was calibrated against shapes of known fractal dimension and it has been found to be accurate and robust with accuracy $\pm 2 \%$.

To determine the algorithmic complexity ("randomness") of the microvascular patterns, relative Lempel-Ziv, L-Z, values were calculated according to the Kaspar and Schuster algorithm using the Chaos Data Analyzer version 2.1 software package (CDA; Pro, Academic Software Library, North Carolina State University, USA), as published elsewhere by one of us [5]. Briefly, vascular network lattices from $251 * 251$ pixel window of the original image were transformed into 16,732 points containing onedimensional vectors and each data point was converted into a single binary digit according to whether the design is touched $(=1)$ or not $(=0)(\mathrm{L}-\mathrm{Z}$ measures the irregularity in a given data set, for example, ordered data correspond to $\mathrm{L}-\mathrm{Z}=0$, while completely disordered data show $\mathrm{L}-\mathrm{Z}=1$ ).

To determine the microvascular density, $\mathrm{d}$, the number of pixels of the microvessels' trajectories was counted and expressed as percentage versus the total area (Image Pro-plus 1.3 software).

The vascular pattern analysis was reproducible, with mean intra- and inter-observer coefficient of variation of 3\% and 5\%, respectively. Mann-Whitney test (D and L-Z) and $t$-test (microvessel density) were applied in order to verify significant differences between the groups.

In RTT, the microvascular pattern appeared less ordered, more irregular or chaotic, than in healthy subjects and some capillaries appeared giant or dilated, ramified and bushy capillaries were observed (Fig. 2).

In RTT, D and L-Z were found significantly higher than in healthy individuals (median (min value - max value): Rett, $\mathrm{D}=1.36$ (1.14-1.66); controls, $\mathrm{D}=1.18$ (0.96-1.33); $p<0.001)$ (Fig. 3); Rett, $\mathrm{L}-\mathrm{Z}=0.32$ (0.20-0.45); controls, $\mathrm{L}-\mathrm{Z}=0.14(0.08-0.24) ; p<0.001)$ (Fig. 4).

In RTT, microvacular density, $d$, resulted higher than in controls (mean \pm SD: Rett, $d=7.6 \% \pm 1.5 \%$; controls, $\mathrm{d}=5.2 \% \pm 1.6 \% ; p<0.01)$.
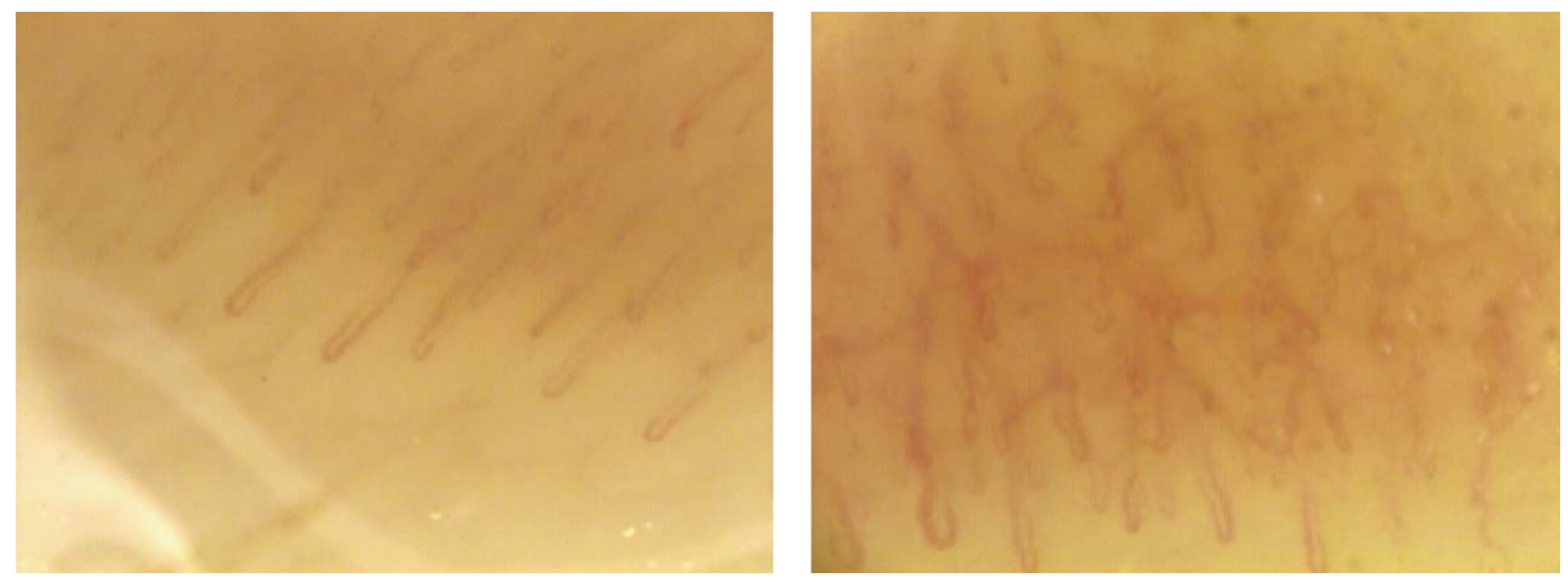

Fig. 2. Microvascular pattern in a control subject is ordered, with capillaries presenting regular distribution and shape (left), in RTT the microvascular pattern is less ordered, more irregular, chaotic in appearance. Some capillaries are giant, dilated and ramified. Videocapillaroscopy, $\times 200$. 


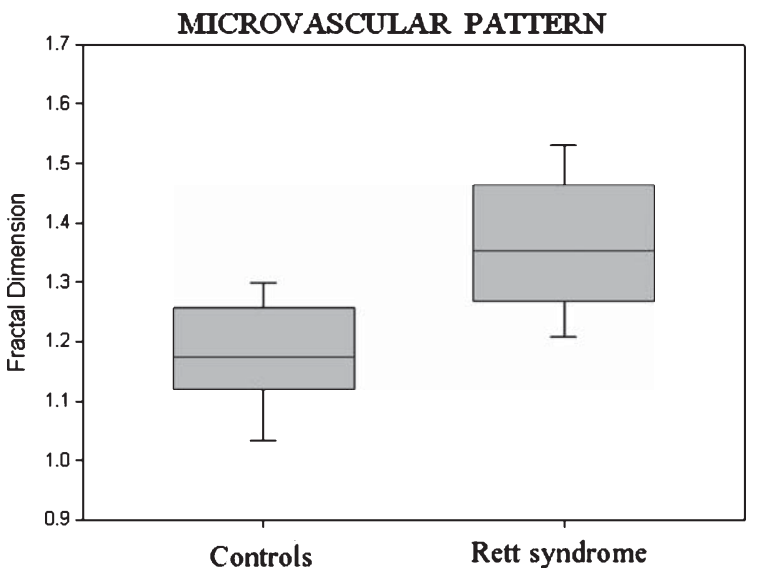

Fig. 3. Box Plot, median + outliers (5th and 95th percentile). Fractal Dimension, Controls vs. Rett syndrome, $p<0.001$.

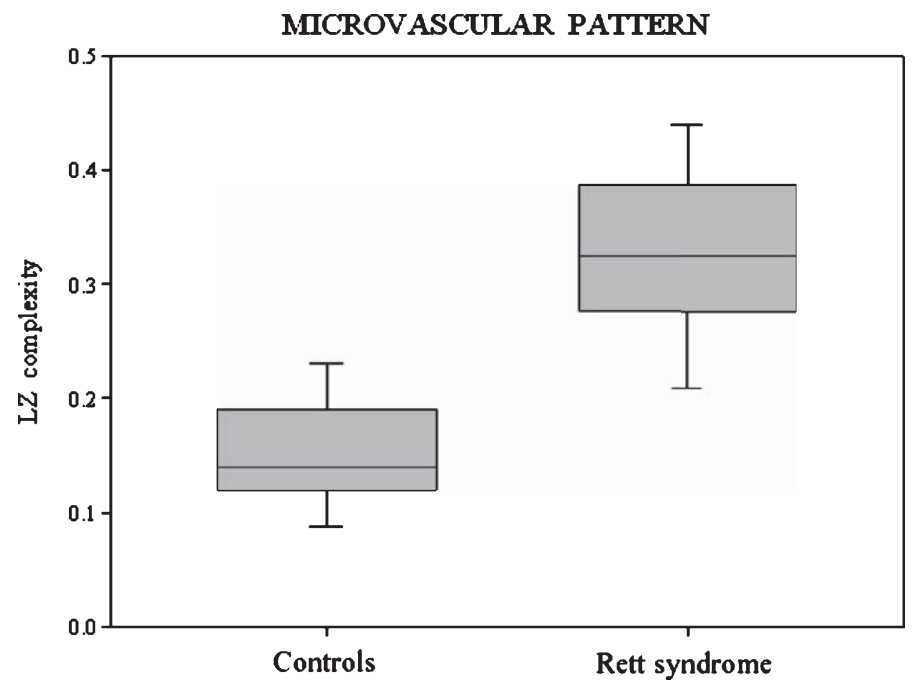

Fig. 4. Box Plot, median + outliers (5th and 95th percentile). Lempel-Ziv complexity, Controls vs. Rett syndrome, $p<0.001$.

The results of the present study indicate the presence of previously unrecognized microvascular abnormalities in Rett syndrome patients. In particular, in RTT we have observed the presence of dilated, ramified and bushy capillaries (characteristic feature of neo-angiogenesis) and microvascular patterns showed fractal dimension, $\mathrm{L}-\mathrm{Z}$ value and density higher than in age-matched healthy individuals.

High fractal dimension, D, an index of geometrical complexity, or, more precisely, of the degree to which the pattern fills the metric space in which it is embedded [8], indicates a high complex microvascular pattern; high L-Z index, an index related to algorithmic complexity, indicates the presence of a relatively high degree of randomness. In other words, fractal analysis shows a microvascular pattern less ordered in RTT than in healthy age-matched controls.

Possible changes of the extracellular matrix (ECM) in RTT should be investigated: in patients with extracellular matrix modifications, microvascular pattern changes have been frequently observed [5] and a close relationship between the ECM and blood vessel network geometry has been reported [11]. 
In 1990, Dieler R. et al. described the presence of amorphous and membranous electron-dense deposits in capillaries of conjunctiva, muscle, and peripheral arteries [7]. Recently, in RTT a modification of perfusion index, attributed to altered peripheral vasoconstriction and intravascular volume status, was reported [6]. Now, our findings demonstrates that in RTT the microvascular pattern is significantly more complex, less ordered and denser than in age-matched controls, with the presence of atypical morphological features. In conclusion, in Rett syndrome, a cohort of subtle modifications of the microvascular system is present: an aspect that deserves to be investigated in depth.

\section{References}

[1] M. Acampa and F. Guideri, Cardiac disease and Rett syndrome, Arch Dis Child 91 (2006), 440-443.

[2] M. Acampa and F. Guideri, Autonomic nervous system alterations in Rett syndrome, Curr Pediatr Rev 5 (2009), 160-168.

[3] R.E. Amir, I.B. Van den Veyver, C.Q. Wan, M. Tran, U. Francke and H.Y. Zoghbu, Rett syndrome is caused by mutations in X-linked MECP2, encoding methyl-CpG-binding protein 2, Nature Genet 23 (1999), 185-188.

[4] A. Banerjee, J. Castro and M. Sur, Rett Syndrome: Genes, Synapses, Circuits, and Therapeutics. Front Psychiatry 3 (2012), $1-13$.

[5] G. Bianciardi, C. Traversi, R. Cattaneo, et al., Phase transition of the Microvascular Network Architecture in Human Pathologies, Theor Biol Forum 105 (2012), 37-45.

[6] C. De Felice, S. Maffei, C. Signorini, et al., Subclinical myocardial dysfunction in Rett syndrome, Eur Heart J Cardiovasc Imaging 13 (2012), 339-345.

[7] R. Dieler, J.M. Schröder and K. Reddemann, Electron-dense lipidic capillary deposits in Rett syndrome, Acta Neuropathol 79 (1990), 573-578.

[8] K. Falconer, Fractal geometry: Mathematical Foundations and Applications, John Wiley, Chichester, 1990.

[9] F. Hanefeld, The spectrum of phenotypes in females with Rett Syndrome, Brain \& Development 25 (2003), 346-351.

[10] P.O. Julu, A.M. Kerr, F. Apartopoulos, et al., Characterization of breathing and associated central autonomic dysfunction in the Rett disorder, Arch Dis Child 85 (2001), 29-37.

[11] N. Suwa, Supracellular structural principle and geometry of blood vessels, Virchows Arch A 390 (1981), 161-179

[12] S.L. Williamson and J. Christodoulou, Rett syndrome: New clinical and molecular insights, Eur J Hum Genet 14 (2006), 896-903. 